\title{
AP-1-mediated chromatin looping regulates ZEB2 transcription: new insights into TNFa-induced epithelial-mesenchymal transition in triple-negative breast cancer
}

\author{
Yichun Qiao ${ }^{1}$, Chiou-Nan Shiue ${ }^{2,3}$, Jian Zhu ${ }^{1}$, Ting Zhuang ${ }^{1}$, Philip Jonsson ${ }^{4}$, \\ Anthony P.H. Wright ${ }^{2}$, Chunyan Zhao ${ }^{1}$, Karin Dahlman-Wright ${ }^{1,5}$ \\ ${ }^{1}$ Department of Biosciences and Nutrition, Novum, Karolinska Institutet, Huddinge, Sweden \\ ${ }^{2}$ Clinical Research Center (KFC), Department of Laboratory Medicine, Karolinska Institutet, Huddinge, Sweden \\ ${ }^{3}$ Department of Pediatrics, Hualien Tzu Chi Hospital, Buddist Tzu Chi Medical Foundation, Hualien, Taiwan \\ ${ }^{4}$ Center for Nuclear Receptors and Cell Signaling, Department of Biology and Biochemistry, University of Houston, \\ Houston, USA \\ ${ }^{5}$ Science for Life Laboratory, Karolinska Institutet, Solna, Sweden \\ Correspondence to: \\ Chunyan Zhao, e-mail: chunyan.zhao@ki.se \\ Karin Dahlman-Wright, e-mail: karin.dahlman-wright@ki.se \\ Keywords: AP-1, triple-negative breast cancer, ZEB2, epithelial-mesenchymal transition, TNFa \\ Received: October 28, $2014 \quad$ Accepted: January 16, 2015 Published: January 30, 2015
}

\section{ABSTRACT}

The molecular determinants of malignant cell behaviour in triple-negative breast cancer (TNBC) are poorly understood. Recent studies have shown that regulators of epithelial-mesenchymal transition (EMT) are potential therapeutic targets for TNBC. In this study, we demonstrate that the inflammatory cytokine TNFa induces EMT in TNBC cells via activation of AP-1 signaling and subsequently induces expression of the EMT regulator ZEB2. We also show that TNFa activates both the PI3K/Akt and MAPK/ERK pathways, which act upstream of AP-1. We further investigated in detail AP-1 regulation of ZEB2 expression. We show that two ZEB2 transcripts derived from distinct promoters are both expressed in breast cancer cell lines and breast tumor samples. Using the chromosome conformation capture assay, we demonstrate that AP-1, when activated by TNFa, binds to a site in promoter 1b of the ZEB2 gene where it regulates the expression of both promoter $1 \mathrm{~b}$ and $1 \mathrm{a}$, the latter via mediating long range chromatin interactions. Overall, this work provides a plausible mechanism for inflammation-induced metastatic potential in TNBC, involving a novel regulatory mechanism governing ZEB2 isoform expression.

\section{INTRODUCTION}

Triple-negative breast cancer (TNBC), a tumor type defined by lack of expression of the estrogen, progesterone and HER 2 receptors, represents a more aggressive and metastatic form of breast cancer than other types of breast cancer [1]. Furthermore, treatment of patients with TNBC is challenging. Whilst breast tumors that express estrogen receptor (ER) are responsive to ER antagonists and aromatase inhibitors and HER2-positive tumors are effectively treated with HER2-blocking antibodies and/or HER2 kinase inhibitors [2], TNBC lacks a targeted therapy. Understanding the molecular pathways determinant for TNBC behaviour is one strategy for developing more effective treatments for TNBCs.

The transcription factor AP-1 consists of different dimeric combinations of either homodimers of Jun proteins (c-Jun, JunB and JunD) or heterodimers of Jun and Fos proteins (c-Fos, FosB, Fra-1 and Fra-2). AP-1 regulates the expression of AP-1 target genes after binding to $12-O$-tetradecanoylphorbol-13-acetate (TPA) response elements (TREs) associated with regulated genes [3]. Upon activation by growth factors, hormones or cytokines, AP-1 signaling plays a critical role in numerous cellular processes, including proliferation, differentiation, apoptosis, cell migration, and transformation [4]. 
$\mathrm{TNF} \alpha$, one of the inflammatory cytokines, signals via TNF receptors, TNFRI and TNFRII. Increasing evidence indicates that a chronic and consistent expression of TNF $\alpha$ in tumors promotes tumor development and progression [5]. Specifically in relation to breast cancer, $\mathrm{TNF} \alpha$ was found to be highly expressed in breast tumors as compared with normal tissues [6], and patients with more advanced breast tumor phenotypes were shown to have elevated TNF $\alpha$ serum levels $[6,7]$. TNF $\alpha$ promotes breast cancer cell proliferation and migration in vitro [8] and contributes to mammary tumorigenesis in vivo [9]. In addition, depletion of TNF $\alpha$ expression in a TNBC cell line led to apoptosis and inhibition of cell proliferation, indicating that TNF $\alpha$ plays a fundamental role in the promotion and progression of TNBC [10].

ZEB2 belongs to a small family of transcriptional factors characterized by containing a homeo domain flanked by two separated zinc finger clusters [11]. It is expressed in various types of human tumors, such as breast cancer, gastric cancer, and ovarian cancer [11]. ZEB2 is a potent repressor of E-cadherin through its direct binding to the E-cadherin promoter and a key player in tumor cell invasion and metastasis $[12,13]$. Consequently, understanding the structure and the regulation of the ZEB2 gene is critical.

Gene looping is increasingly recognized to play important regulatory roles in gene expression [14]. The use of recently developed techniques such as chromosome conformation capture $(3 \mathrm{C})$ has revealed that higher-order chromatin structure involves long-range loop formation between distant genomic elements [15]. Long-range interactions between promoters and distal elements have been discovered in a wide variety of gene loci, and the formation of looping interactions is significantly correlated with gene expression [16].

Epithelial-to-mesenchymal transition (EMT) is a cellular process critical to normal morphogenesis but also cancer metastasis [17-19]. EMT can be triggered by different signals received from tumor microenvironments, such as TNF $\alpha$, TGF $\beta$, EGF, WNTs and Notch [18-20]. During the EMT, epithelial cells acquire fibroblast-like properties and show reduced intercellular adhesion and enhanced motility [21]. One of the hallmarks of EMT is loss of expression of the cell-cell junction protein E-cadherin [17-19]. Several transcription factors, including Snail, Slug, ZEB1, Twist, and ZEB2, have been shown to act as master regulators of the EMT program [22-24]. We have recently reported that AP-1 promotes cell invasion through transcriptional upregulation of ZEB2 in TNBC cells [13]. In this study, we further dissect the AP-1-ZEB2 axis in TNF $\alpha$-induced EMT in TNBC cells.

\section{RESULTS}

\section{TNFa induces EMT in TNBC cells}

EMT is characterized by down-regulation of epithelial markers such as E-cadherin and up-regulation of mesenchymal markers such as N-cadherin and fibronectin. Figure 1A shows that the TNBC cell lines BT549 and Hs578T acquired EMT-like morphological features, such as a spindle-shaped appearance, in response to $\mathrm{TNF} \alpha$ treatment. In agreement with the change in cellular appearance, TNF $\alpha$ treatment led to significant reduction in E-cadherin protein expression as well as increases in $\mathrm{N}$-cadherin and fibronectin protein expression (Figure 1A), all characteristics of EMT.

\section{TNFa-mediated EMT in TNBC cells is dependent on AP-1-ZEB2 signaling}

We have previously demonstrated that AP-1 signaling, via activation of ZEB2, induces cell invasion of TNBC cells [13]. We have also demonstrated that Fra-1 and c-Jun are highly expressed AP-1 protein family members in these cells [13]. In the following set of experiments, we explored whether the AP-1-ZEB2 axis also mediates TNF $\alpha$-induced EMT in TNBC cells.

We depleted Fra-1 or c-Jun (Figure 1B) and ZEB2 (Figure 1C) in BT549 cells, and analysed cellular appearance and EMT markers in TNF $\alpha$-stimulated cells. Whereas control cells underwent marked EMT after 3 days of TNF $\alpha$ treatment, Fra-1- or c-Jun-depleted cells (Figure 1B) and ZEB2-depleted cells (Figure 1C) did not acquire EMT characteristics in the form of spindle-like morphological features. Similar results were obtained with an independent pool of Fra-1, c-Jun or ZEB2 siRNAs (data not shown), suggesting that this effect is specific to Fra-1, c-Jun and ZEB2 silencing. Consistent with the observed effect on morphological features, Fra-1, c-Jun, or ZEB2 knockdown increased the expression level of E-cadherin, and decreased the expression levels of $\mathrm{N}$-cadherin and fibronectin (Figure 1B and 1C). Combined, these data suggest that the AP-1-ZEB2 axis is required for TNF $\alpha$ mediated EMT in TNBC cells.

\section{TNFa induces AP-1 activation and ZEB2 expression in TNBC cells}

We have previously demonstrated that AP-1 activation downstream of the PI3K/Akt and MAPK/ERK signaling pathways transactivates ZEB2 gene expression [13]. To gain additional insight into the molecular details of the AP-1-ZEB2 axis in TNF $\alpha$-induced EMT in TNBC cells and in particular, if our previous finding regarding AP-1 activation of ZEB2 can be translated to the effects of $\mathrm{TNF} \alpha$ in this system, we assayed AP-1 activation and ZEB2 expression in response to TNF $\alpha$ treatment in TNBC cells.

As shown in Figure 2A, TNF $\alpha$ treatment markedly up-regulated the levels of phospho-Fra-1, Fra-1, phosphoc-Jun, and c-Jun proteins at 2, 4 and 6 hours. To determine whether the PI3K/Akt and MAPK/ERK signaling pathways mediate TNF $\alpha$ up-regulation of AP-1 levels and activity, we used two pharmacological inhibitors, LY294002 
A
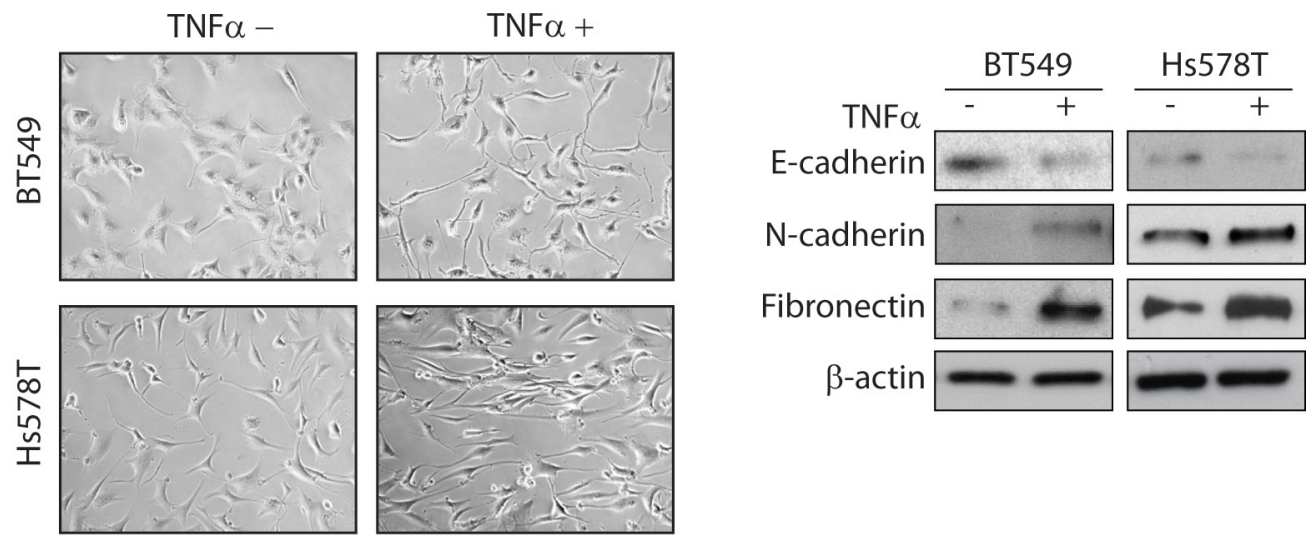

B
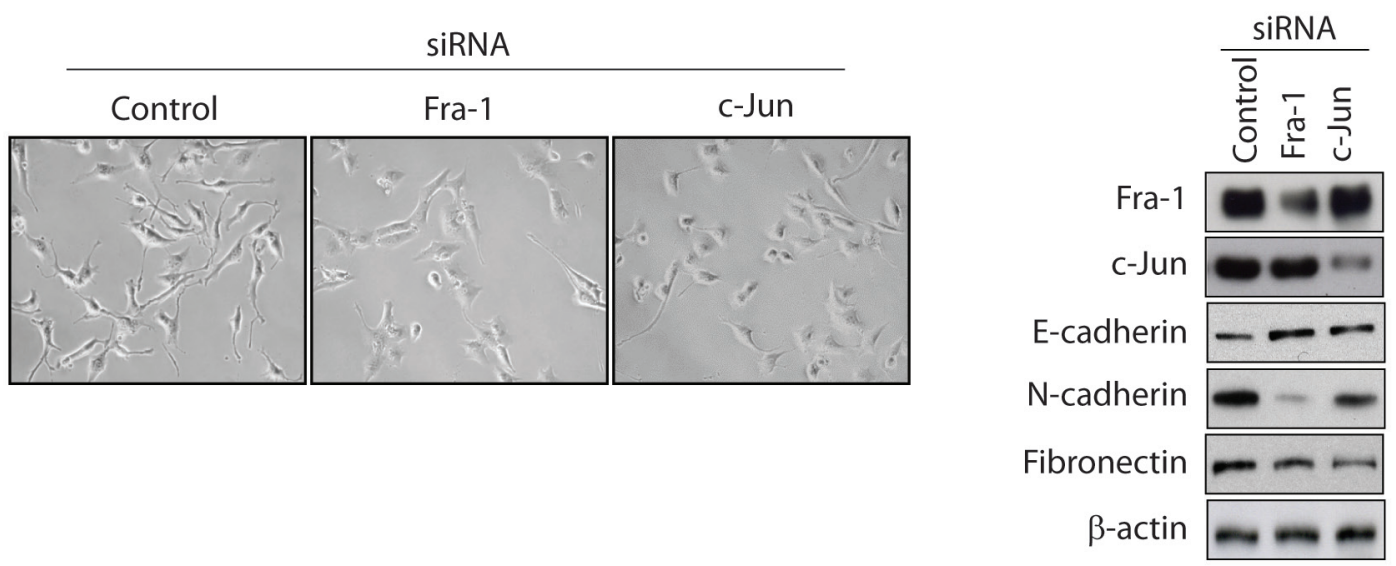

C
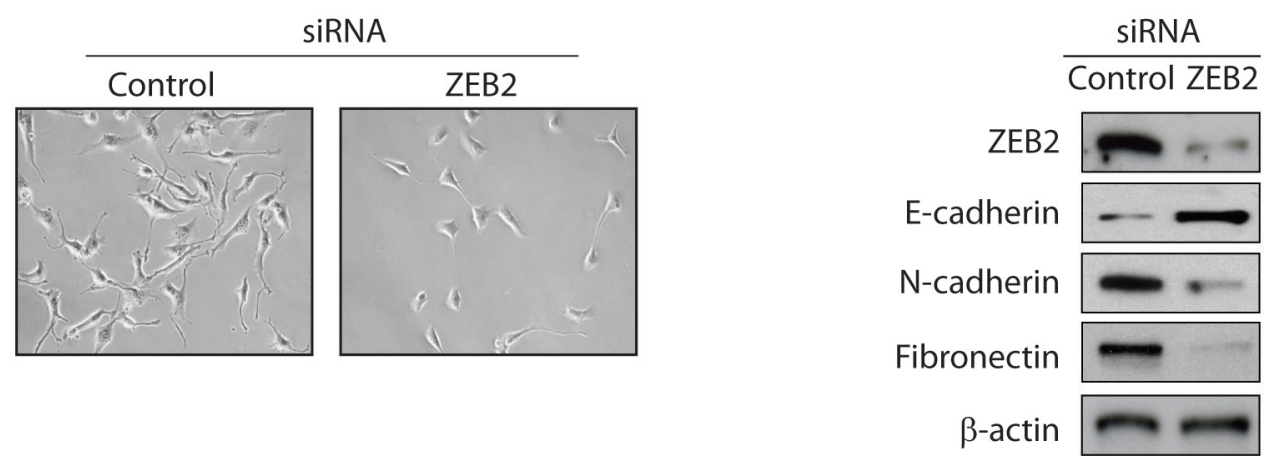

Figure 1: TNFa-mediated EMT in TNBC cells is dependent on AP-1-ZEB2 signaling. (A) BT549 and Hs578T cells were serum-starved overnight and then treated with or without TNF $\alpha(10 \mathrm{ng} / \mathrm{ml})$ for 72 hours. The cells were examined by phase contrast microscopy and Western blots analysis for epithelial (E-cadherin) and mesenchymal (N-cadherin and fibronectin) markers. $\beta$-actin was used as a loading control. Representative images from two independent studies. (B) BT549 cells transfected with control, Fra-1 or c-Jun siRNA were treated as above. Western blot confirms knockdown of Fra-1 or c-Jun levels. (C) BT549 cells transfected with control or ZEB2 siRNA were treated as above. Western blot confirms knockdown of ZEB2 levels. The same image for control siRNA is shown for Figure 1B and Figure $1 \mathrm{C}$ because all conditions were assessed in the same experiment. 
and PD98059, to block the PI3K/Akt and MAPK/ ERK pathways, respectively. We found that LY294002 and PD98059 in TNF $\alpha$-stimulated cells inhibited TNF $\alpha$-induced expression of Fra-1 and c-Jun proteins (Figure 2B), suggesting that activation of the PI3K/Akt and MAPK/ERK pathways is responsible for TNF $\alpha$-induced AP-1 protein expression. The effect of inhibitor treatment on these pathways was confirmed by assessing the level of Akt and ERK1/2 phosphorylation (Figure 2B). We next examined ZEB2 expression after TNF $\alpha$ stimulation. Compared to untreated cells, TNF $\alpha$ significantly increased ZEB2 mRNA levels after 2 and 4 hours of treatment in the BT549 and Hs578T cell lines with a subsequent increase in ZEB2 protein levels (Figure 2C). Finally, a role of AP-1 signaling in mediating $\mathrm{TNF} \alpha$-induced up-regulation of ZEB2 was provided by depleting Fra-1 or c-Jun in BT549 cells prior to TNF $\alpha$ stimulation. Depletion of Fra-1 or c-Jun markedly impaired the induction of ZEB2 gene expression by TNF $\alpha$ (Figure 2D). Furthermore, TNF $\alpha$ treatment increased binding of Fra-1 and c-Jun to an AP-1 binding region in an intron of the $Z E B 2$ gene that we have previously described [13] (Figure 2E). To further determine whether phospho-Fra-1 is binding to DNA, we assessed the ability of phospho-Fra-1 to bind to the ZEB2 gene. Consistent with the markedly elevated levels of phosphoFra-1 upon TNF $\alpha$ treatment, phospho-Fra-1 binding was increased by TNF $\alpha$ (Figure 2F). Collectively, these results show that TNF $\alpha$-induced AP-1 activation, via distinct pathways, is a critical mediator of ZEB2 up-regulation in TNBC cells.

\section{TNFa regulates $\mathrm{ZEB} 2$ via two promoters dependent on AP-1 in TNBC cells}

As ZEB2 participates in regulation of EMT, a detailed knowledge of mechanisms for its expression is of fundamental importance. Human ZEB2 mRNAs initiate from two distinct first exons, referred to as exon 1a and exon $1 \mathrm{~b}$, respectively, which are located $2.2 \mathrm{~kb}$ apart and are spliced to the common exons 2-10 (Figure 3A). We examined the expression of exon $1 \mathrm{a}$ and exon $1 \mathrm{~b}$ transcripts in a panel of breast cancer cell lines and primary human breast tumors. As shown in Figure 3B, among 11 breast cancer cell lines, expression of the two transcripts was detected in 5 cell lines. In primary breast tumors, the exon 1a transcript was detected in all analyzed samples, whilst the exon $1 \mathrm{~b}$ transcript displayed a more restricted expression (Figure 3C). Together, these results reveal that two alternatively spliced 5'UTR isoforms of the ZEB2 gene can be expressed in breast cancer cell lines and breast cancer tissues, indicating that the two promoters, driving the respective transcripts, are functional.

Using ChIP-seq, we have previously found that the ZEB2 gene harbors only one AP-1 binding site that is located in the promoter $1 \mathrm{~b}$ region (Figure $3 \mathrm{~A}$ ) and based on this, we hypothesized that AP-1 regulation of ZEB2 is restricted to the ZEB2 exon $1 \mathrm{~b}$ transcript. However, as shown in Figure 3D, expression of transcripts including exon 1a as well as expression of transcripts including exon $1 \mathrm{~b}$ were decreased significantly after the knockdown of Fra-1 or c-Jun (Figure 3D). Furthermore, $\mathrm{TNF} \alpha$ treatment increased both exon $1 \mathrm{a}$ and $1 \mathrm{~b}$ transcript levels (Figure 3E). Moreover, depletion of Fra-1 or c-Jun markedly impaired the TNF $\alpha$-mediated induction of both transcripts (Figure 3E). Combined, these results indicate that $\mathrm{TNF} \alpha$ regulates $\mathrm{ZEB} 2$ via two promoters dependent on AP-1 in TNBC cells.

\section{AP-1-mediated chromatin looping regulates ZEB2 transcription}

To investigate whether AP-1 binding to promoter $1 \mathrm{~b}$ of the $Z E B 2$ gene also regulates the activity of promoter 1a by long range chromosomal interactions, we performed a $3 \mathrm{C}$ assay to determine whether there is a physical proximity between the ZEB2 promoters $1 \mathrm{a}$ and $1 \mathrm{~b}$ (Figure $4 \mathrm{~A}$ ). PCR bands resulting from the ligation products indicated that the promoter $1 \mathrm{~b}$ was in close proximity to promoter 1a (Figure 4B). Negative control samples either from non-crosslinked chromatin with ligation by T4 ligase or from crosslinked chromatin without subsequent ligation did not generate any PCR bands. These results clearly demonstrate that promoters $1 \mathrm{a}$ and $1 \mathrm{~b}$ of the $Z E B 2$ gene are in close proximity via chromatin looping. To further evaluate the role of AP-1 as a direct regulator of the loop formation between the two promoter regions, the ChIP-loop technique was used to determine whether AP-1 is physically associated with the gene loop. Our results demonstrated an association of AP-1 with the ZEB2 gene loops (Figure 4C).

To address whether AP-1 is required for the gene looping within the $Z E B 2$ gene, we determined the effect of AP-1 depletion on the promoter $1 \mathrm{a}$ and $1 \mathrm{~b}$ interaction. Western blot analysis confirmed that siRNA targeted to Fra-1 or c-Jun efficiently reduced the expression of Fra-1 or c-Jun (Figure 4D). 3C assays revealed that knockdown of Fra-1 or c-Jun impaired gene loop formation (Figure 4D), indicating that AP-1 is a critical mediator of long-distance interaction between the $1 \mathrm{a}$ and $1 \mathrm{~b}$ promoters. Finally, treatment of cells with TNF $\alpha$ increased long-range interaction between the $1 \mathrm{a}$ and $1 \mathrm{~b}$ promoters (Figure 4E), which correlated with TNF $\alpha$-mediated induction in the mRNA levels of the exon $1 \mathrm{a}$ and $1 \mathrm{~b}$ containing transcripts (Figure 3E).

Together, these results clearly demonstrated that AP-1, when activated by $\mathrm{TNF} \alpha$, binds to a site in the promoter $1 \mathrm{~b}$ region of the $Z E B 2$ gene from which it regulates the expression of both promoter $1 \mathrm{~b}$ and promoter $1 \mathrm{a}$, the latter via mediating long-range chromatin interactions. 
A

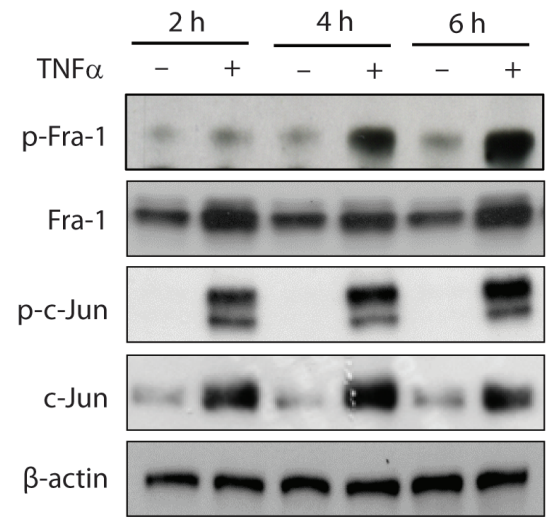

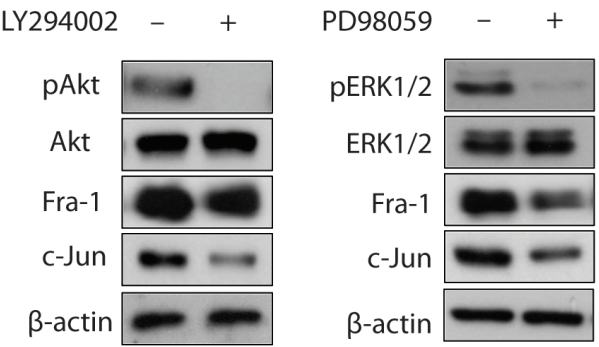

C

ZEB2 qPCR/BT549

ZEB2 qPCR/Hs578T
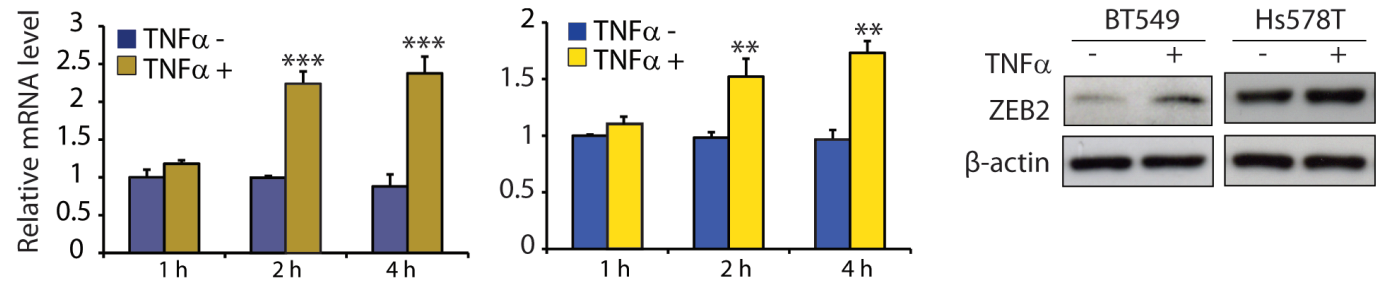

D

E

F
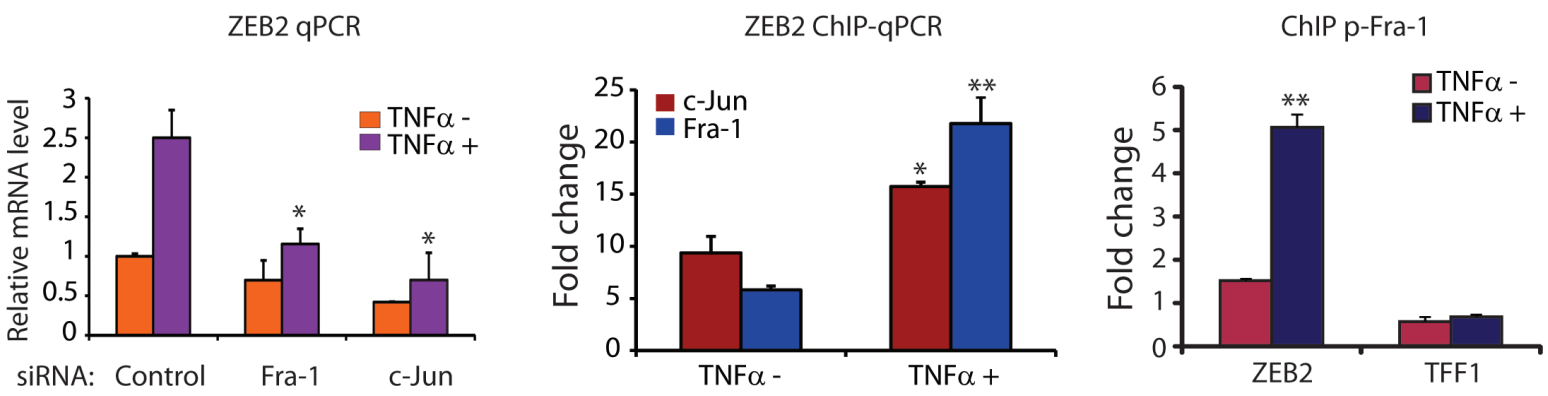

Figure 2: TNFa induces AP-1 activation and ZEB2 expression in TNBC cells. (A) Western blot analysis of p-Fra-1, Fra-1, p-c-Jun, c-Jun, and $\beta$-actin (as a loading control) in BT549 cells treated with or without TNF $\alpha$ (10 ng/ml) for 2, 4 and 6 hours. (B) TNF $\alpha$ mediates Fra-1 and c-Jun expression via the PI3K/Akt and MAPK/ERK pathways. Western blot analysis of pAkt, Akt, pERK1/2, ERK1/2, Fra-1, c-Jun and $\beta$-actin (as a loading control) in BT549 cells pretreated with LY294002 (25 $\mu \mathrm{M})$ or PD98059 (25 $\mu$ M) for 6 hours, followed by stimulation with TNF $\alpha(10 \mathrm{ng} / \mathrm{ml})$ for 6 hours. (C) TNF $\alpha$ induces ZEB2 expression at both the mRNA and protein levels in BT549 and Hs578T cells. ZEB2 mRNA levels were determined by qPCR after treatment with or without TNF $\alpha(10 \mathrm{ng} / \mathrm{ml})$ for the indicated times. Data are shown as means with SD. ${ }^{* *} p<0.01,{ }^{* * *} p<0.001$ compared with TNF $\alpha$ minus $(n=3)$. The protein levels of ZEB2 after 6 hours of treatment with TNF $\alpha$ were analyzed by Western blot. $\beta$-actin was used as a loading control. (D) Depletion of Fra-1 or c-Jun markedly impaired the induction of ZEB2 gene expression by TNF $\alpha$. Values are mean $\pm \mathrm{SD}(n=3) . * p<0.05$ compared with control siRNA. (E) ChIP-qPCR analysis showing increased binding of Fra-1 and c-Jun to ZEB2 following TNF $\alpha$ treatment. Columns, mean fold enrichment of Fra-1 or c-Jun relative to IgG; bars, $\mathrm{SD}(n=3)$. ${ }^{*} p<0.05,{ }^{* *} p<0.01$ compared with TNF $\alpha$ minus. (F) ChIP-qPCR analysis showing increased binding of phospho-Fra-1 to ZEB2 following TNF $\alpha$ treatment. TFF1 is used as a negative control. ChIP was performed with antibody against phospho-Fra-1. Columns, mean fold enrichment of phospho-Fra-1 relative to IgG; bars, $\mathrm{SD}(n=3)$. ${ }^{* *} p<0.01$ compared with TNF $\alpha$ minus. 
A

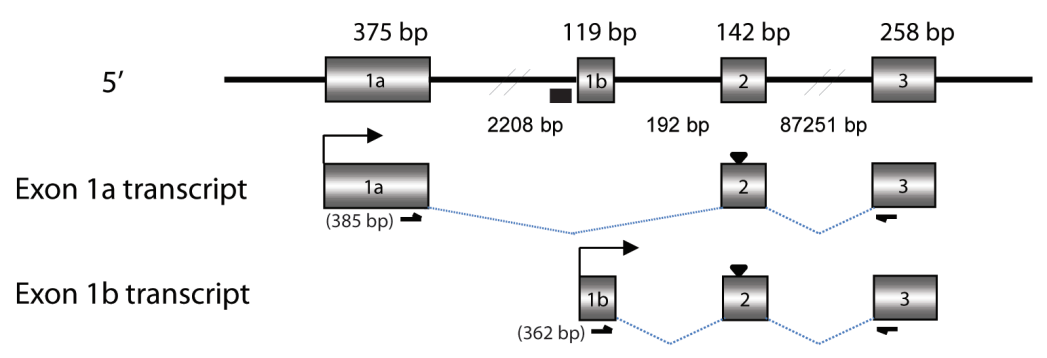

B

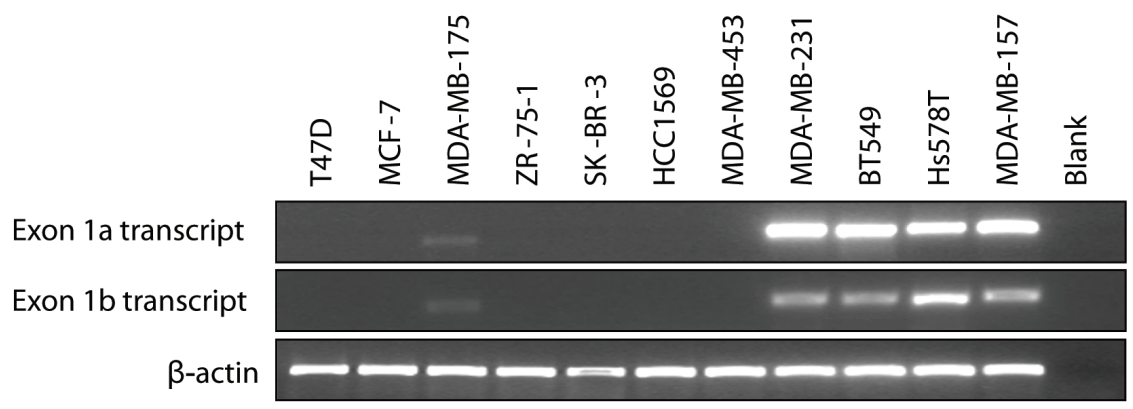

C

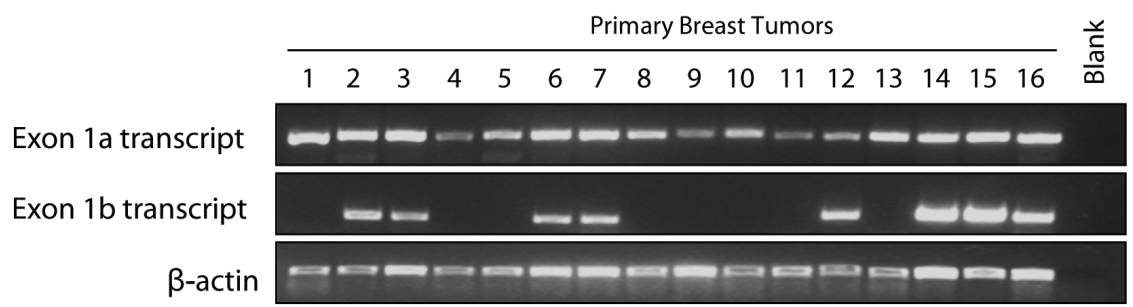

$\mathrm{D}$

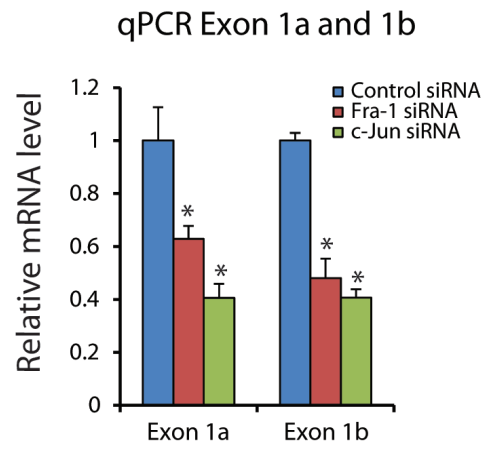

E

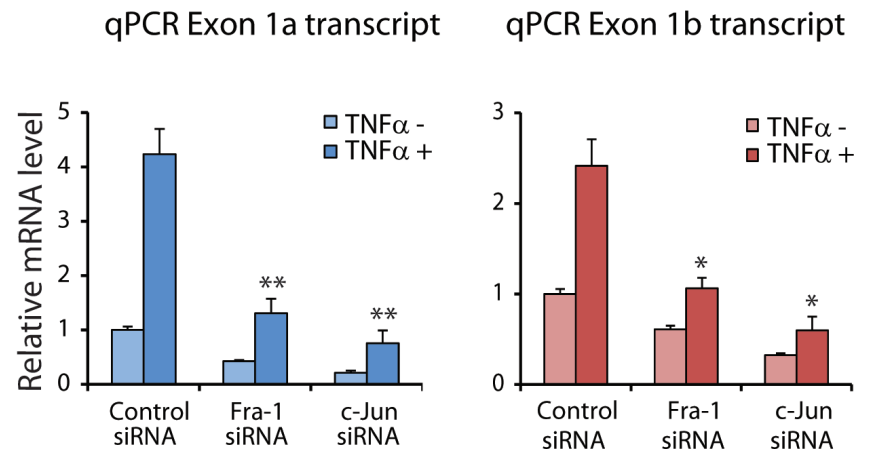

Figure 3: TNFa regulates ZEB2 via two promoters dependent on AP-1 in TNBC cells. (A) Structure of the 5' region of the ZEB2 gene and schematic of mRNA initiated from two distinct first exons, named exon 1a and exon 1b, splicing to downstream exon 2 . Origins of sequences: exon 1a was from ENST00000558170, and exon 1b from ENST00000409487. The size of each exon and distances between exons are indicated. ' $\boldsymbol{\nabla}$ ' at exon 2 indicates ATG, ' $\boldsymbol{\square}$ ' indicates AP-1 binding site and bent arrows indicate transcriptional start sites. RT-PCR primer sets to amplify exon 1a and exon $1 \mathrm{~b}$ transcripts are also indicated. Expected sizes of the products are shown in the parentheses. (B) RT-PCR detection of the expression of exon 1a and exon 1b transcripts in RNA isolated from breast cancer cell lines. Expression of $\beta$-actin mRNA was used as an internal control. (C) RT-PCR detection of the expression of exon 1a and exon $1 \mathrm{~b}$ transcripts in breast tumor RNA. (D) qPCR data showing decreased exon 1a and exon $1 \mathrm{~b}$ transcript levels in BT549 cells upon Fra-1 or c-Jun depletion. Values are mean $\pm \mathrm{SD}(n=3) .{ }^{*} p<0.05$ compared with control siRNA. (E) qPCR data showing that depletion of Fra-1 or c-Jun markedly impaired the induction of exon 1a and exon $1 \mathrm{~b}$ transcript expression by TNF $\alpha$. Values are mean $\pm \operatorname{SD}(n=3) .{ }^{*} p<0.05,{ }^{* *} p<0.01$ compared with control siRNA. 
A

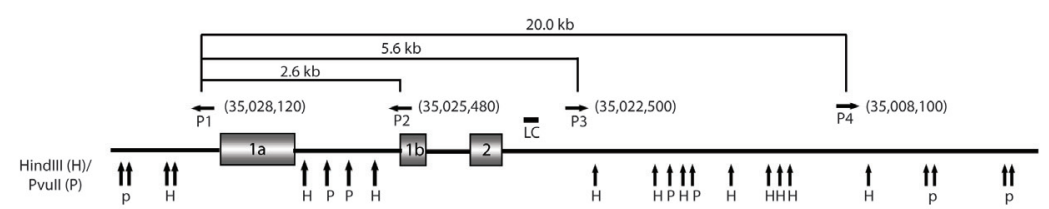

B

c

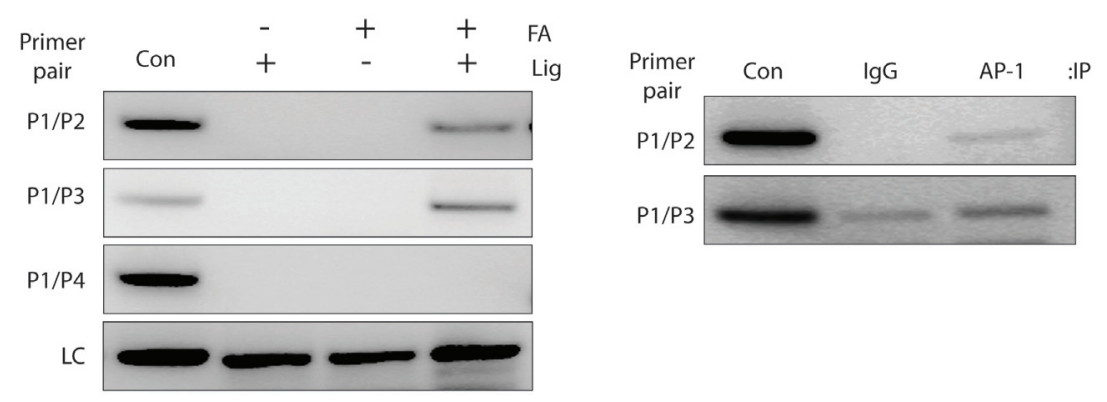

D

$\mathrm{E}$
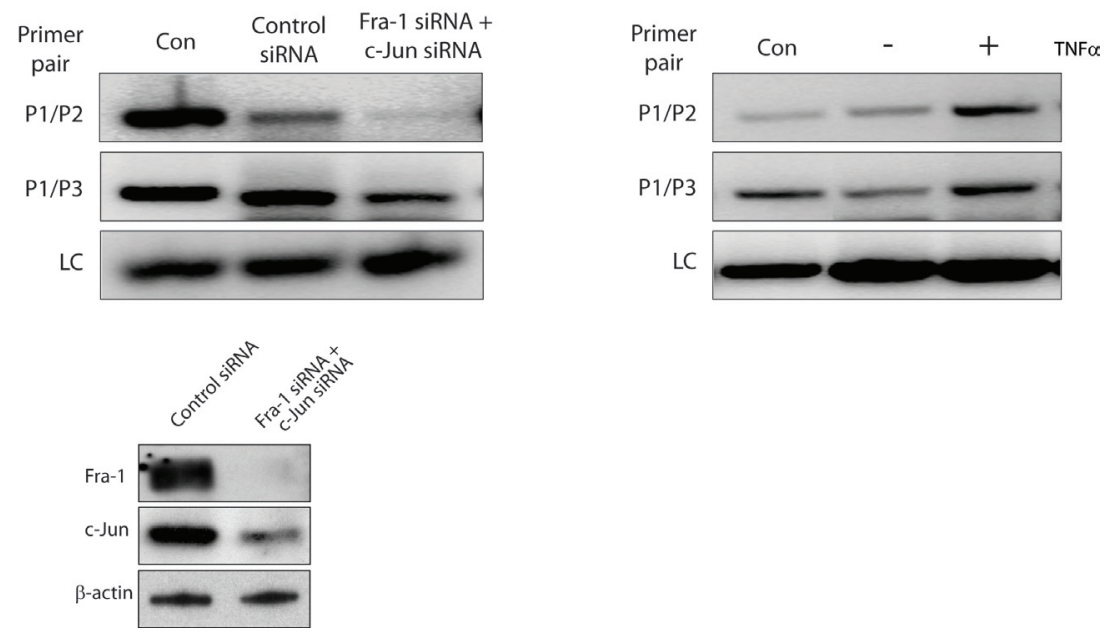

Figure 4: AP-1-mediated chromatin looping regulates ZEB2 transcription. (A) Schematic of the ZEB2 locus, showing $13833 \mathrm{~kb}$ region from base 34891668 to 35030000 (GenBank accession no. NW_004929304). Vertical arrows represent the locations of cleavage sites for HindIII $(\mathrm{H})$ and PvuII $(\mathrm{P})$. Horizontal arrows indicate locations and orientations of the combinations of primer sets used in 3C assay. Numbers in brackets indicate primer positions (numbering according to GenBank accession no. NW_004929304). Primer sequences were as follows: P1, GAGAGCATGATATTAAACGGCATGGGGTCTAAGTTTTGGA; P2, GGAGAATCCTGAAAACTCGGTGGAGGTTGGGAAGATGCTG;P3,TCTCTGAGTAATTTAGTAGACACTAAAATTACACCTAATG; P4, CGATCTTTGAATTTATCTGCAATGAGGTACAGCTTTAATG. The region amplified as a loading control (LC) is also indicated. (B) A looped topological conformation of the ZEB2 promoter 1a and 1b is observed in BT549 cells. Results of 3C analysis with the indicated primer pairs on chromatin prepared from BT549 cells by crosslinking or not with formaldehyde (FA), and digestion with combination of HindIII and PvuII, followed by ligation (Lig) or not. PCR products from two primer pairs P1/P2 and P1/P3, which are closely located to exon 1a or exon $1 \mathrm{~b}$, were detected in samples from crosslinked chromatin with subsequent ligation (lane 4). As a negative control, primer pair P1/P4 generated no PCR product (panel 3). Lane Con shows positive control fragment, which indicates the expected size of PCR products in the $3 \mathrm{C}$ assay if the tested DNA ends are in close proximity. The sequences of primers for amplifying size marker templates will be provided on request. The loading control (LC) for each lane is shown in the bottom panel. (C) AP-1 associates with the juxtaposed promoter 1a and 1b. ChIP-loop analysis of putative gene loop region in chromatin prepared from BT549 cells using antibodies against IgG and AP-1 (a pool of Fra-1 and c-Jun antibodies). (D) Knockdown of AP-1 abolishes gene looping of promoter 1a and 1b. 3C assays performed on chromatin prepared from BT549 cells after transfection with control siRNA or siRNAs against Fra-1 and c-Jun for 72 hours. Bottom panels, Western blot analysis showing reduced protein levels of Fra-1 and c-Jun 72 hours after siRNA transfection in BT549 cells, as determined by using an anti-Fra-1 or anti-c-Jun antibody, respectively. $\beta$-actin was used as a loading control. (E) TNF $\alpha$ treatment increases gene looping. 3C assays performed on chromatin prepared from serum-starved BT549 cells treated with or without TNF $\alpha$ for 2 hours. 


\section{DISCUSSION}

It has become increasingly clear that inflammatory cytokines such as TNF $\alpha$ facilitate both tumor development and metastatic progression [25]. TNF $\alpha$ is a major inflammatory cytokine shown to be highly expressed in breast carcinomas [6]. TNFaexpression is also associated with tumor metastasis of breast cancer [26]. Our study provides several insights into the regulation of inflammatory cytokine-induced EMT that contributes to increases in invasive behavior of TNBC cells. We report that $\mathrm{TNF} \alpha$-induced EMT in TNBC cells functions via activation of AP-1 signaling, which in turn induces expression of the EMT regulator ZEB2. We also show that TNF $\alpha$ activates both the PI3K/Akt and MAPK/ERK pathways, which act upstream of AP-1. Moreover, we dissect mechanisms regulating expression of the ZEB2 gene, whose expression is shown to be associated with AP-1-driven long-range combinatorial regulation.

Compared to other breast tumor subtypes, the PI3K/ AKT pathway is enhanced in TNBC, and plays a critical role in promoting EMT and invasion in various cancers [27]. Furthermore, AP-1 components can be activated by MAPK/ERK signaling pathway [28]. In this study, we demonstrate that these two signaling pathways are upstream regulators of AP- 1 in response to TNF $\alpha$. We find that LY294002 and PD98059 treatment led to a significant down-regulation of Fraland c-Jun proteins, suggesting that the activation of the PI3K/Akt and MAPK/ERK pathways is responsible for $\mathrm{TNF} \alpha$-mediated AP-1 up-regulation in TNBC cells.

Our study reveals a critical role for AP-1-ZEB2 axis in TNF $\alpha$-initiated EMT. Although previous research has implicated the interplay between inflammatory cytokines and the activation of different signaling pathways, like Wnt, Notch and NF-kB signaling in the regulation of EMT-like processes [29], our results further suggest that AP-1 signaling is necessary for $\mathrm{TNF} \alpha$-induced EMT. The inflammatory mediators, such as TNF $\alpha, \mathrm{TGF} \beta$ and IL-6, in regulation of Snail have been implicated in the initiation of EMT [30-32]. It has been demonstrated that Snail enhances TNF $\alpha$-induced cancer cell migration and invasion in breast cancer cells, through stabilization of $\mathrm{NF}-\kappa \mathrm{B}$ activation. [32]. However, much less is known about the regulation of the other regulators. Consistent with our data on the role of ZEB2 in TNF $\alpha$-induced EMT, Vandewalle et al. [33] showed that exogenous ZEB2 expression in a colon cancer cell line induced a dramatic morphological conversion, from an epithelial cell state to a fibroblast-like phenotype. Expanding on our previous findings that ZEB2 caused an invasive phenotype in TNBC cells [13], this study indicates that ZEB2 induction mediated by the inflammatory cytokine TNF $\alpha$ is critical for EMT and thus provides a plausible molecular mechanism contributing to TNBC aggressiveness.
The human ZEB2 gene includes two promoters followed by distinct non-coding first exons, which are located $2.2 \mathrm{~kb}$ apart and are spliced to the common exon 2 containing the translation initiation site. This study analyzed for the first time the distribution of the two transcripts of ZEB2 directed from distinct promoters in a number of primary breast tumors as well as a number of cell lines. Both transcripts were highly expressed in TNBC cells such as MDA-MB-231, BT549, Hs578T and MDAMB-157, representing the invasive breast cancer subtype. This result is in agreement with our previous findings that high levels of a total pool of ZEB2 mRNAs were detected only in TNBC cell lines [13]. In relevance to these data, it has been reported that ZEB2 expression is higher in various cancers compared with normal tissues [34, 35]. We showed that transcripts from both promoters were expressed in breast cancer tissues, indicating that both promoters are targets for regulation of ZEB2 expression in breast cancer. Interestingly, we found that the exon 1a transcript was detected in all analyzed breast tumors samples, whilst exon $1 \mathrm{~b}$ transcript displayed a more restricted expression. This may be associated with distinct breast cancer subtypes included in the study. Many genes are known to be expressed with alternative 5'UTRs often originating from distinct promoters. $5^{\prime} \mathrm{UTR}$ regions can influence mRNA stability and translational efficiency and thus differential expression of transcripts with different $5^{\prime} \mathrm{UTRs}$ is a means to modulate protein expression [36]. The potential significance of the expression of alternative UTRs is related to tissue-specific expression patterns and a contribution of dysregulated expression of alternative UTRs to the development of disease [36]. The role of the two ZEB2 promoters on human tissue-specific expression patterns and ZEB2 protein expression levels needs to be further investigated.

It is increasingly recognized that the expression of multiple genes may be coordinately regulated through chromatin looping [37]. In this study, we observed that AP-1 could regulate expression of two distinct ZEB2 mRNAs, which differ in their 5'UTR but translate the same protein, through the use of alternative promoters by in vivo long-range chromosomal interactions. However, only one transcript variant has an AP-1 binding site in its promoter region, raising the important question of whether binding of AP-1 to the AP-1 element located in the proximal promoter may act long-range to activate distal promoter through DNA looping. We used the $3 \mathrm{C}$ assay to demonstrate the existence of AP-1-dependent in vivo long-range chromosomal interactions between ZEB2 distal and proximal promoters. Moreover, knockdown of AP-1 abolished chromatin loop formation. Thus, our data provide a novel mechanism of AP-1-driven longrange combinatorial regulation. Such chromatin-based transcription mechanisms have been reported for several genes. For example, Gong et al. [38] have shown that 
BCL2 gene expression is controlled by chromatin looping between a 3'-UTR region and BCL2 promoter.

We present evidence for chromatin looping between the ZEB2 promoters $1 \mathrm{a}$ and $1 \mathrm{~b}$ (located $\sim 2.4 \mathrm{~kb}$ apart). DNA looping over $\sim 100 \mathrm{bp}$ to $10 \mathrm{~kb}$ distances that bring regulatory elements into the proximity of promoters, contributing to gene activation, has been documented for many genes, including the CD68 gene spanning over a $2.5 \mathrm{~kb}$ distance [39], the HO-1 gene over a $6.0 \mathrm{~kb}$ distance [40], and the insulin gene spanning a region of $1.4 \mathrm{~kb}$ [41]. More recently, comprehensive chromatin interactions were mapped in human cells using a genome-wide $3 \mathrm{C}$ analysis method (Hi-C). It was demonstrated that the sizes of the identified interacting DNA loci range from several hundred bp to over $50 \mathrm{~kb}$, with a median of $10.5 \mathrm{~kb}$ [14], further emphasizing the occurrence of looping interactions over variable distances.

In the present work, we demonstrate that the proinflammatory cytokine TNF $\alpha$-triggered EMT of TNBC cells is mediated through AP-1-induced ZEB2 upregulation. We dissect mechanisms underlying regulation of the ZEB2 gene, whose expression is shown to be associated with AP-1-driven long-range combinatorial regulation. Our study not only reveals a critical mechanism underlying inflammation-induced metastatic potential in TNBC but also provides evidence of AP-1 and its upstream ERK and Akt signaling, and ZEB2 as potential drug targets for reversing EMT, the key event in the acquisition of invasive potential.

\section{METHODS}

\section{Cell lines and reagents}

T47D，MCF-7，MDA-MB-175，ZR-75-1，SKBR-3, HCC1569, MDA-MB-453, MDA-MB-231, BT549, Hs578T, and MDA-MB-157 cells were obtained from American Type Culture Collection. Human recombinant TNF $\alpha$ was purchased from Roche, and LY294002 and PD98059 were from Sigma.

\section{Reverse transcription-PCR}

Reverse transcription-PCR (RT-PCR) was done using standard procedures. The primers used in each reaction were as follows: Exon 1a ZEB2 transcript, forward 5'-TTCTCACCATTTCTGGCCAAA-3' and reverse 5'-CACCCTCCCTTATTTCATCTTCCT-3'; Exon 1b ZEB2 transcript, forward 5'-GGGTGCAACACACCAAACAA-3' and reverse 5'-CACCCTCCCTTATTTCATCTTCCT-3'; $\beta$-actin, forward 5'-CAAGGCCAACCGCGAGAAGAT-3' and reverse 5'-GTCCCGGCCAGCCAGGTCCAG.

\section{Human breast tumor samples}

The 16 breast tumor samples in this study have been previously described [42]. The studies were approved by the ethical committee of the Karolinska Institutet.

\section{Chromatin immunoprecipitation}

BT549 cells were cultured on $150 \mathrm{~mm}$ dishes and were starved in RPMI 1640 medium containing 1\% fetal bovine serum (FBS) overnight and then treated with or without TNF $\alpha(10 \mathrm{ng} / \mathrm{ml})$ for 2 hours. Chromatin immunoprecipitation (ChIP) was performed as previously described [42]. The following antibodies were used: anti-p-Fra-1 (Ser265; \#3880) from Cell Signaling Technology; anti-Fra-1 (R-20), anti-c-Jun (H-79) and normal rabbit IgG from Santa Cruz Biotechnology. The immunoprecipitated DNA was amplified by qPCR using primers to a known AP-1 binding region in the ZEB2 promoter $1 \mathrm{~b}$. Primer sequences used were: forward 5'-TGACCGTATGAGGGAATGCA-3' and reverse 5'-CCG CCTAGCCCTGAGTCAA-3'

\section{Western blotting}

Western blot analyses were performed as previously described [42]. Antibodies used were anti-Fra-1 (R-20; sc-605), anti-c-Jun (H-79; sc-1694), anti-p-c-Jun (KM1; sc-822) and anti-ZEB2 (E-11; sc-271984) from Santa Cruz Biotechnology; anti-E-cadherin (610182), anti-Ncadherin (610920), and anti-fibronectin (610077) from BD Biosciences; anti- $\beta$-actin from Sigma; anti-p-Fra-1 (Ser265; \#3880), anti-Akt \#9272, anti-phospho-Akt (Ser473; \#9271), anti-ERK, and anti-phospho-ERK were from Cell Signaling Technology.

\section{qPCR}

qPCR was performed in a 7500 Fast Real-Time PCR (Applied Biosystems, Foster City, CA) with Fast SYBRGreen Master mix (Applied Biosystems) according to conditions specified by the manufacturer. The specificity of all primer pairs was checked with melting curve analysis. Primer sequences will be provided on request.

\section{RNA interference}

BT549 cells were cultured to $50 \%$ confluency and transfected with Fra-1 ON-TARGET plus SMARTpool (Thermo Scientific; L-004341-00-0010), c-Jun ONTARGET plus SMARTpool (Thermo Scientific; L-003268-00-0010), ZEB2 ON-TARGET plus SMARTpool (Thermo Scientific; L-006914-02-0010) or nontargeting pool (Thermo Scientific; D-001810-1020) using the INTERFERin siRNA transfection reagent (Polyplus) according to the manufacturer's instructions.

\section{C}

3C assays were performed in BT549 cells as described previously [43]. Briefly, BT549 cells were formaldehyde cross-linked at room temperature for $10 \mathrm{~min}$. After the addition of glycine for $5 \mathrm{~min}$, cells were pelleted 
and intact nuclei were resuspended in lysis buffer (SigmaAldrich Sweden AB) containing protease inhibitors (Roche Diagnostics GmbH, Mannheim, Germany). Appropriate amounts of the chromatin solutions were subjected to overnight digestion with HindIII and PvuII (New England Biolabs, Hitchin, UK). After ligation by T4 ligase, crosslinking was reversed and DNA extracted by phenol/chloroform before precipitation by ethanol. ChIPloop was carried out as described [43]. The crosslinked chromatin fragments were immunoprecipitated with $\mathrm{IgG}$ or a pool of anti-Fra-1 and anti-c-Jun antibodies.

\section{ACKNOWLEDGMENTS}

This work was supported by scholarship from the China Scholarship Council and grants from the Swedish Cancer Society (Cancerfonden) and the Center for Biosciences. The authors acknowledge the Bioinformatic and Expression Analysis core facility at the Karolinska Institute (BEA, www.bea.ki.se) for performing the Affymetrix assays. We are grateful to Lars-Arne Haldosen for critically reading the manuscript.

\section{CONFLICTS OF INTEREST}

No potential conflicts of interest were disclosed.

\section{Abbreviations}

TNBC - triple-negative breast cancer, EMT epithelial-mesenchymal transition, ER - estrogen receptor, $3 \mathrm{C}$ - chromosome conformation capture, ChIP - Chromatin immunoprecipitation.

\section{REFERENCES}

1. Elias AD. Triple-negative breast cancer: a short review. Am J Clin Oncol. 2010; 33:637-645.

2. Pal SK, Childs BH, Pegram M. Triple negative breast cancer: unmet medical needs. Breast Cancer Res Treat. 2011; 125:627-636.

3. Shaulian E, Karin M. AP-1 as a regulator of cell life and death. Nat Cell Biol. 2002; 4:E131-136.

4. Shaulian E. AP-1-The Jun proteins: oncogenes or tumor suppressors in disguise? Cell Signal. 2010; 22:894-899.

5. Bertazza L, Mocellin S. The dual role of tumor necrosis factor (TNF) in cancer biology. Curr Med Chem. 2010; 17:3337-3352.

6. Soria G, Ofri-Shahak M, Haas I, Yaal-Hahoshen N, Leider-Trejo L, Leibovich-Rivkin T, Weitzenfeld P, Meshel T, Shabtai E, Gutman M, Ben-Baruch A. Inflammatory mediators in breast cancer: coordinated expression of TNFalpha \& IL-1beta with CCL2 \& CCL5 and effects on epithelial-to-mesenchymal transition. BMC Cancer. 2011; 11:130.
7. Garcia-Tunon I, Ricote M, Ruiz A, Fraile B, Paniagua R, Royuela M. Role of tumor necrosis factor-alpha and its receptors in human benign breast lesions and tumors (in situ and infiltrative). Cancer Sci. 2006; 97:1044-1049.

8. Rivas MA, Carnevale RP, Proietti CJ, Rosemblit C, Beguelin W, Salatino M, Charreau EH, Frahm I, Sapia S, Brouckaert P, Elizalde PV, Schillaci R. TNF alpha acting on TNFR1 promotes breast cancer growth via p42/P44 MAPK, JNK, Akt and NF-kappa B-dependent pathways. Exp Cell Res. 2008; 314:509-529.

9. Warren MA, Shoemaker SF, Shealy DJ, Bshar W, Ip MM. Tumor necrosis factor deficiency inhibits mammary tumorigenesis and a tumor necrosis factor neutralizing antibody decreases mammary tumor growth in neu/erbB2 transgenic mice. Mol Cancer Ther. 2009; 8:2655-2663.

10. Pileczki V, Braicu C, Gherman CD, Berindan-Neagoe I. TNF-alpha gene knockout in triple negative breast cancer cell line induces apoptosis. Int J Mol Sci. 2012; 14:411-420.

11. Katoh M. Integrative genomic analyses of ZEB2: Transcriptional regulation of ZEB2 based on SMADs, ETS1, HIF1alpha, POU/OCT, and NF-kappaB. Int J Oncol. 2009; 34:1737-1742.

12. Nam EH, Lee Y, Park YK, Lee JW, Kim S. ZEB2 upregulates integrin alpha5 expression through cooperation with Sp1 to induce invasion during epithelial-mesenchymal transition of human cancer cells. Carcinogenesis. 2012; 33:563-571.

13. Zhao C, Qiao Y, Jonsson P, Wang J, Xu L, Rouhi P, Sinha I, Cao Y, Williams C, Dahlman-Wright K. Genome-wide profiling of AP-1-regulated transcription provides insights into the invasiveness of triple-negative breast cancer. Cancer Res. 2014; 74:3983-3994.

14. Jin F, Li Y, Dixon JR, Selvaraj S, Ye Z, Lee AY, Yen CA, Schmitt AD, Espinoza CA, Ren B. A high-resolution map of the three-dimensional chromatin interactome in human cells. Nature. 2013; 503:290-294.

15. Sajan SA, Hawkins RD. Methods for identifying higherorder chromatin structure. Annu Rev Genomics Hum Genet. 2012; 13:59-82.

16. Sanyal A, Lajoie BR, Jain G, Dekker J. The long-range interaction landscape of gene promoters. Nature. 2012; 489:109-113.

17. Lee JM, Dedhar S, Kalluri R, Thompson EW. The epithelialmesenchymal transition: new insights in signaling, development, and disease. J Cell Biol. 2006; 172:973-981.

18. Thiery JP, Acloque H, Huang RY, Nieto MA. Epithelialmesenchymal transitions in development and disease. Cell. 2009; 139:871-890.

19. Yang J, Weinberg RA. Epithelial-mesenchymal transition: at the crossroads of development and tumor metastasis. Dev Cell. 2008; 14:818-829.

20. Polyak K, Weinberg RA. Transitions between epithelial and mesenchymal states: acquisition of malignant and stem cell traits. Nat Rev Cancer. 2009; 9:265-273. 
21. Thiery JP, Sleeman JP. Complex networks orchestrate epithelial-mesenchymal transitions. Nat Rev Mol Cell Biol. 2006; 7:131-142.

22. Medici D, Hay ED, Olsen BR. Snail and slug promote epithelial-mesenchymal transition through beta-catenin-Tcell factor-4-dependent expression of transforming growth factor-beta3. Mol Biol Cell. 2008; 19:4875-4887.

23. Wellner U, Schubert J, Burk UC, Schmalhofer O, Zhu F, Sonntag A, Waldvogel B, Vannier C, Darling D, zur Hausen A, Brunton VG, Morton J, Sansom O, Schuler J, Stemmler MP, Herzberger C, et al. The EMT-activator ZEB1 promotes tumorigenicity by repressing stemnessinhibiting microRNAs. Nat Cell Biol. 2009; 11:1487-1495.

24. Yang J, Mani SA, Donaher JL, Ramaswamy S, Itzykson RA, Come C, Savagner P, Gitelman I, Richardson A, Weinberg RA. Twist, a master regulator of morphogenesis, plays an essential role in tumor metastasis. Cell. 2004; 117:927-939.

25. Balkwill F. Tumour necrosis factor and cancer. Nat Rev Cancer. 2009; 9:361-371.

26. Weitzenfeld P, Meron N, Leibovich-Rivkin T, Meshel T, Ben-Baruch A. Progression of luminal breast tumors is promoted by menage a trois between the inflammatory cytokine TNFalpha and the hormonal and growth-supporting arms of the tumor microenvironment. Mediators Inflamm. 2013; 2013:720536.

27. Umemura S, Yoshida S, Ohta Y, Naito K, Osamura RY, Tokuda Y. Increased phosphorylation of Akt in triplenegative breast cancers. Cancer Sci. 2007; 98:1889-1892.

28. Doehn U, Hauge C, Frank SR, Jensen CJ, Duda K, Nielsen JV, Cohen MS, Johansen JV, Winther BR, Lund LR, Winther O, Taunton J, Hansen SH, Frodin M. RSK is a principal effector of the RAS-ERK pathway for eliciting a coordinate promotile/invasive gene program and phenotype in epithelial cells. Mol Cell. 2009; 35:511-522.

29. Lamouille S, Xu J, Derynck R. Molecular mechanisms of epithelial-mesenchymal transition. Nat Rev Mol Cell Biol. 2014; 15:178-196.

30. Liu J, Hu G, Chen D, Gong AY, Soori GS, Dobleman TJ, Chen XM. Suppression of SCARA5 by Snail1 is essential for EMT-associated cell migration of A549 cells. Oncogenesis. 2013; 2:e73.

31. Sullivan NJ, Sasser AK, Axel AE, Vesuna F, Raman V, Ramirez N, Oberyszyn TM, Hall BM. Interleukin-6 induces an epithelial-mesenchymal transition phenotype in human breast cancer cells. Oncogene. 2009; 28:2940-2947.

32. Wu Y, Deng J, Rychahou PG, Qiu S, Evers BM, Zhou BP. Stabilization of snail by NF-kappaB is required for inflammation-induced cell migration and invasion. Cancer Cell. 2009; 15:416-428.
33. Vandewalle C, Comijn J, De Craene B, Vermassen P, Bruyneel E, Andersen H, Tulchinsky E, Van Roy F, Berx G. SIP1/ZEB2 induces EMT by repressing genes of different epithelial cell-cell junctions. Nucleic Acids Res. 2005; 33:6566-6578.

34. Jacob S, Nayak S, Fernandes G, Barai RS, Menon S, Chaudhari UK, Kholkute SD, Sachdeva G. Androgen receptor as a regulator of ZEB2 expression and its implications in epithelial-to-mesenchymal transition in prostate cancer. Endocr Relat Cancer. 2014; 21:473-486.

35. Kahlert C, Lahes S, Radhakrishnan P, Dutta S, Mogler C, Herpel E, Brand K, Steinert G, Schneider M, Mollenhauer M, Reissfelder C, Klupp F, Fritzmann J, Wunder C, Benner A, Kloor M, et al. Overexpression of ZEB2 at the invasion front of colorectal cancer is an independent prognostic marker and regulates tumor invasion in vitro. Clin Cancer Res. 2011; 17:7654-7663.

36. Hughes TA. Regulation of gene expression by alternative untranslated regions. Trends Genet. 2006; 22:119-122.

37. Kadauke S, Blobel GA. Chromatin loops in gene regulation. Biochim Biophys Acta. 2009; 1789:17-25.

38. Gong F, Sun L, Wang Z, Shi J, Li W, Wang S, Han X, Sun Y. The BCL2 gene is regulated by a special AT-rich sequence binding protein 1-mediated long range chromosomal interaction between the promoter and the distal element located within the 3'-UTR. Nucleic Acids Res. 2011; 39:4640-4652.

39. O'Reilly D, Greaves DR. Cell-type-specific expression of the human CD68 gene is associated with changes in Pol II phosphorylation and short-range intrachromosomal gene looping. Genomics. 2007; 90:407-415.

40. Deshane J, Kim J, Bolisetty S, Hock TD, Hill-Kapturczak $\mathrm{N}$, Agarwal A. Sp1 regulates chromatin looping between an intronic enhancer and distal promoter of the human heme oxygenase-1 gene in renal cells. J Biol Chem. 2010; 285:16476-16486.

41. Babu DA, Chakrabarti SK, Garmey JC, Mirmira RG. Pdx1 and BETA2/NeuroD1 participate in a transcriptional complex that mediates short-range DNA looping at the insulin gene. J Biol Chem. 2008; 283:8164-8172.

42. Zhao C, Matthews J, Tujague M, Wan J, Strom A, Toresson G, Lam EW, Cheng G, Gustafsson JA, DahlmanWright K. Estrogen receptor beta2 negatively regulates the transactivation of estrogen receptor alpha in human breast cancer cells. Cancer Res. 2007; 67:3955-3962.

43. Shiue CN, Berkson RG, Wright AP. c-Myc induces changes in higher order rDNA structure on stimulation of quiescent cells. Oncogene. 2009; 28:1833-1842. 